\title{
A Ruelle Operator for a Real Julia Set
}

\author{
G. M. Levin ${ }^{1, \star}$, M. L. Sodin ${ }^{2}$, and P. M. Yuditski ${ }^{3}$
}

1 Institute of Mathematics, Hebrew University of Jerusalem, 91904, Israel

${ }^{2}$ Mathematical Division of the Institute for Low Temperature Physics and Engineering, Lenin Av. 47, 310164 Kharkov, USSR

${ }^{3}$ Institute for Mechanization and Electrification of Agriculture, Kharkov, USSR

Received December 3, 1990; in revised form March 28, 1991

Abstract. Let $R$ be an expanding rational function with a real bounded Julia set, and let $(L g)(x)=\sum_{R y=x} \frac{g(y)}{\left[R^{\prime}(y)\right]^{2}}$ be a Ruelle operator acting in a space of functions analytic in a neighbourhood of the Julia set. We obtain explicit expressions for the resolvent function $E(x, z ; \lambda)=(I-\lambda L)^{-1} \frac{1}{z-x}$ and, in particular, for the Fredholm determinant $D(\lambda)=\operatorname{det}(I-\lambda L)$. It gives us an equation for calculating the escape rate. We relate our results to orthogonal polynomials with respect to the balanced measure of $R$. Two examples are considered.

\section{Introduction}

The facts from the Fatou-Julia theory of iterations used below are contained, for example, in the surveys of Blanchard [6], and Milnor [15]. We shall use also some notions of the thermodynamic formalism for expanding mappings developed in the works of Sinai, Ruelle and Bowen (e.g. see Bowen [7, Chap. 1,2], and the recent survey of Ruelle [18], which is supplied with an extensive list of references).

Let $R$ be a rational function with a real bounded Julia set $J$. We shall assume that the mapping $R$ is expanding on $J$ (another word: hyperbolic), that is, for some $A>0, c>1$, and all integers $n>0$,

$$
\inf \left\{\left|R_{n}^{\prime}(x)\right|: x \in J\right\} \geqq A c^{n},
$$

where $R_{n}$ is the $n^{\text {th }}$ iteration of $R$ [in the case of real bounded Julia set the inequality (1.1) is equivalent to the conditions: $R$ has not neutral fixed points and critical points on $J$, see Sect. 2.1]. Under these hypotheses $J$ is a Cantor-type set of zero length.

\footnotetext{
* The first named author was sponsored in part by the Landau Center for Research in Mathematical Analysis, supported by the Minerva Foundation (Germany)
} 
In what follows we shall focus basically on the study of the operator

$$
L g(x)=\sum_{R(y)=x} \frac{g(y)}{\left[R^{\prime}(y)\right]^{2}} .
$$

The Ruelle version of the Perron-Frobenius theorem (hereafter called the RPFtheorem) is applied to this operator acting on the space of continuous functions $C(J)$. In particular, the spectral radius $\varrho$ of this operator is the simple eigenvalue of operators $L$ and $L^{*}$, and all other eigenvalues have strictly smaller modules. The eigenfunction $h$ of the operator $L$ corresponding to the eigenvalue $\varrho$ is strictly positive on $J$, and the corresponding eigenmeasure $v$ of operator $L^{*}$ is nonnegative. The measure $h v$ is (up to normalization) the Gibbs state for function $\left|R^{\prime}\right|^{-2}$.

The value $\alpha=\log \frac{1}{\varrho}$ has an important dynamical interpretation: it follows from the Köebe distortion theorem (see e.g. [10]) and the RPF-theorem that $\alpha$ coincides with the "escape rate": $\alpha=\lim _{n \rightarrow \infty} \frac{1}{n} \log \frac{1}{\operatorname{area} \Omega_{n}}$, where $\Omega=\Omega_{0}$ is a neighborhood of $J$ and $\Omega_{n}=R_{-n} \Omega$ its full preimage under the $n$-iteration $R_{n}$. This value has been investigated both numerically and in a series of physical articles (see especially Widom, Bensimon, Kadanoff and Shenker [21] and Kadanoff and Tang [12]).

In the case when $R(z)=z^{2}-p$, the spectral properties of the operator $L$ were used for the study of the convergence of diagonal Padé approximants to the Stieltjes transformation of the balanced measure of $R$ (Levin [14]) and for the investigation of a limit-periodic finite difference operator with the singularly continuous simple spectrum acting on the space $\ell^{2}(\mathbb{Z})$ (Sodin, Yuditski [19]).

Using a general idea of Ruelle we consider the operator $L$ in the space $A(\Omega)$ of functions, which are analytic in a neighborhood $\Omega \supset J$ of the Julia set containing no critical points of the function $R$. In this space the operator $L$ is an integral operator, and the Fredholm-Grothendieck theory is applied to this operator. The operator $L$ has only point spectrum $\left\{\varrho_{k}\right\}_{k=1}^{\infty}$ plus its sole limit point zero, and by virtue of the RPF-theorem, $\varrho=\varrho_{1}$ is, as before, the greatest eigenvalue of the operator $L=\left.L\right|_{A(\Omega)}$.

The present paper is devoted to the constructive investigation of spectral properties of the operator $L_{\infty}$

Let $D(\lambda)=\operatorname{det}(I-\lambda L)=\prod_{n=1}^{\infty}\left(1-\lambda \varrho_{n}\right)$ be the Fredholm determinant of the operator L. According to the definition,

$$
D(\lambda)=\exp \left\{-\sum_{m=1}^{\infty} \frac{\lambda^{m}}{m} \operatorname{tr}\left(L^{m}\right)\right\} .
$$

The traces of the operator $L$ can be calculated very easily in this case (see Sect. 3 ), but the corresponding expansion of $\log D(\lambda)$ converges only in the disk $|\lambda|<\varrho$ and requires the knowledge of the fixed points of all iterations $R_{m}, m=1,2, \ldots$.

In Sect. 4 using perturbation theory we obtain a more convenient expression for $D(\lambda)$, which requires a calculation only of iterations of critical points of $R$. In the case when $R$ is a polynomial, this expression is the Taylor-series expansion of the entire function $D(\lambda)$. In Sect. 5 we find the explicit formula for resolvent

$$
E(x, z ; \lambda)=(I-\lambda L)^{-1} \frac{1}{z-x}=\frac{D(x, z ; \lambda)}{D(\lambda)} .
$$


In the last three sections (6-8) we dwell on two examples: $R(z)=z^{2}-p, p>2$, and $R(z)=\sigma z-\frac{1}{z}, \sigma>1$. In the first example our general formula has the form

$$
D(\lambda)=1+\sum_{n=1}^{\infty} \frac{(\lambda / 2)^{n}}{R(0) \ldots R_{n}(0)}
$$

The entire function $D(\lambda)$ decreases for $\lambda>0$, and the series (1.4) converges very rapidly. This fact is important for calculating the value of the escape rate. Besides, in this case we find the Taylor-series expansion of function $\frac{1}{D(\lambda)}($ Sect. 7).

\section{Preliminaries}

2.1. Let $R$ be an arbitrary rational function with a real bounded Julia set $J$. According to Sullivan's theorem (Sullivan [20]), the domain $G=\mathbb{C} \backslash J$ is either an attractive basin, or a rotation domain (Siegel disk or Herman ring). The latter case is impossible, because the map $R: G \rightarrow G$ has a degree more than one. Thus, $G$ is the attractive domain of a fixed point $a \in \bar{G}$. It follows from this and from the criterion for expansion (e.g. Lyubich [13]) the equivalence of the following conditions in the considered case $J \subset \mathbb{R}$ :

(a) $R$ is expanding on $J$,

(b) there are no critical and neutral fixed points of $R$ on $J$.

2.2. Fix an expanding rational function $R$ with a real bounded Julia set $J$, so that one of the two equivalent conditions (a) or (b) is satisfied, and the domain $G=\mathbb{C} \backslash J$ is the attractive domain of the attracting fixed point $a \in G$.

We may assume $a=\infty$. Then either $\infty$ is an attracting point, and

$$
R(z) \sim \sigma z, \quad \sigma>1, \text { for }|z| \text { large }
$$

or $\infty$ is a superattracting point, and then

$$
R(z) \sim b z^{m}, \quad m \geqq 2, b \neq 0 \text {, for }|z| \text { large. }
$$

By the theorems of Schröder and Böttcher the function $R(z)$ is analytically conjugate in a neighbourhood of infinity to the simplest transformations of the form (2.1) or (2.2). More precise, there exists an analytic function $\varphi(z)$ in a neighborhood of infinity such that

$$
u=\varphi(z)=z+c+\frac{d}{z}+\ldots
$$

and in addition

in the case (2.1), and

$$
\varphi(R(z))=\sigma \varphi(z)
$$

in the case (2.2).

$$
\varphi(\varepsilon R(z))=(\varphi(\varepsilon z))^{m}, \quad \varepsilon^{m-1}=b,
$$

According to these basic functional equations the function $\varphi$ may be extended to an analytic function in the domain $G$ with branching points in the critical points of $R$ and their preimages under the mappings $R_{n}$ for all $n \in \mathbb{N}$.

2.3. Let $\operatorname{crit}(R)$ denote the set of all finite critical points of the expanding function $R$. It is known (e.g. see Hirsch and Pugh [11]), that there exists a Lyapunov metric $\|\cdot\|$ in some neighbourhood $V$ of $J, V \cap \operatorname{crit}(R)=\emptyset$, i.e.

$$
\left\|D_{x} R(v)\right\| \geqq K\|v\|,
$$


for some $K>1$ and for all points $x \in V$ and all tangent vectors $v$ at point $x$. Let $\Omega \subset V$ be $\delta$-neighbourhood of $J$ with respect to the Lyapunov metric ( $\delta$ is positive and small).

Then

$$
\overline{R^{-1}(\Omega)} \subset \Omega
$$

(see, for example, Milnor [15]).

For every smooth contour $\gamma \subset \Omega$, which is close enough to the boundary $\partial \Omega$ and surrounds $J$, we get

$$
J \subset R^{-1}\left(\Omega_{\gamma}\right) \subset \Omega_{\gamma},
$$

where $\Omega_{\gamma}$ is a finite domain bounded by $\gamma$. If now $g \in A(\Omega)$, then by the Cauchy theorem,

$$
L g(z)=\frac{1}{2 \pi i} \int_{\gamma} \frac{g(\tau) d \tau}{R^{\prime}(\tau)[R(\tau)-z]},
$$

where $\gamma$ is such a contour, and $z \in \Omega_{\gamma}$.

2.4. Later on we use the adjoint space of analytic functionals $A^{*}(\Omega)$, which can be identified with the space of functions analytic outside of $\Omega$ and equal to zero at infinity. In other words, if $\tilde{f} \in A^{*}(\Omega)$, then there exist a domain $\Omega_{f} \supset \mathbb{C} \backslash \Omega$ and a function $f \in A_{0}\left(\Omega_{f}\right)$ [it means that $f$ is analytic in $\Omega_{f}$ and $f(\infty)=0$ ] such that

$$
f[g]=\frac{1}{2 \pi i} \int_{\gamma} f(\tau) g(\tau) d \tau,
$$

where $g \in A(\Omega)$ and a contour $\gamma$ seperates singularities of functions $f$ and $g$ and lies in their common domain of holomorphicity. In particular, $f(z)=\widetilde{f}\left[\frac{1}{z-\cdot}\right]$.

2.5. We find a form of the adjoint operator $L^{*}$ acting in the space $A^{*}(\Omega)$. We have:

$$
\begin{aligned}
\left(L^{*} f\right)(z) & =f\left[\left(L \frac{1}{z-\cdot}\right)(\zeta)\right]=f\left[\frac{1}{2 \pi i} \int_{\gamma} \frac{1}{R^{\prime}(\tau)[R(\tau)-\zeta]} \frac{d \tau}{z-\tau}\right] \\
& =\frac{1}{2 \pi i} \int_{\gamma} \frac{f(R(\tau))}{R^{\prime}(\tau)(z-\tau)} d \tau .
\end{aligned}
$$

Applying the Residue Theorem to the exterior of the contour $\gamma$ we obtain:

$$
\left(L^{*} f\right)(z)=\frac{f(R(z))}{R^{\prime}(z)}-\sum_{c \in \operatorname{crit}(R)} \operatorname{Res}_{\tau=\mathrm{c}} \frac{f(R(\tau))}{R^{\prime}(\tau)(z-\tau)} .
$$

Thus, in this situation the passage to the adjoint operator is the passage from an operator on analytic functions in a neighborhood of the repeller $J$ to an operator on functions analytic in a neighborhood of the attracting point $a=\infty$.

\section{Calculation of $\operatorname{Traces} \operatorname{tr}\left(L^{m}\right)$}

Let us use the expression (2.5) to get:

$$
\left(L^{m} g\right)(x)=\frac{1}{2 \pi i} \int_{\gamma_{m}} \frac{g(\tau) d \tau}{R_{m}^{\prime}(\tau)\left[R_{m}(\tau)-x\right]}, \quad g \in A\left(\Omega_{m}\right),
$$


where $\Omega_{m}=R_{-m} \Omega, \Omega_{m} \cap \operatorname{crit}\left(R_{m}\right)=\emptyset, \gamma_{m}=R_{-m} \gamma$. Hence, denoting by fix $\left(R_{m}\right)$ the set of fixed points of $R_{m}$ not equal to $\infty$ (i.e. lying in the Julia set), we obtain

$$
\begin{aligned}
\operatorname{tr}\left(L^{m}\right) & =\frac{1}{2 \pi i} \int_{\gamma_{m}} \frac{d \tau}{R_{m}^{\prime}(\tau)\left[R_{m}(\tau)-\tau\right]}=\sum_{x \in \mathrm{fix}\left(R_{m}\right)} \frac{1}{R_{m}^{\prime}(x)\left[R_{m}^{\prime}(x)-1\right]} \\
& =\sum_{x \in \mathrm{fix}\left(R_{m}\right)} \frac{1}{R_{m}^{\prime}(x)-1}-\sum_{x \in \mathrm{fix}\left(R_{m}\right)} \frac{1}{R_{m}^{\prime}(x)} .
\end{aligned}
$$

The first sum is equal to the residue of the function $\frac{1}{R_{m}(z)-z}$ at infinity, i.e.

$$
\sum_{x \in \text { fix }\left(R_{m}\right)} \frac{1}{R_{m}^{\prime}(x)-1}=\frac{1}{\sigma^{m}-1}
$$

in the case (2.1) and is equal to zero in the case (2.2). These cases can be united into one case, if we let $\sigma=\infty$ for the superattracting point.

Substituting (3.1) and (3.2) into the expression (1.3) for the Fredholm determinant, we obtain

$$
\begin{aligned}
D(\lambda) & =\exp \left\{-\sum_{m=1}^{\infty} \frac{\lambda^{m}}{m\left(\sigma^{m}-1\right)}\right\} \exp \left\{\sum_{m=1}^{\infty} \frac{\lambda^{m}}{m} \sum_{x \in \mathrm{fix}\left(R_{m}\right)} \frac{1}{R_{m}^{\prime}(x)}\right\} \\
& =\prod_{n=1}^{\infty}\left(1-\frac{\lambda}{\sigma^{n}}\right) \exp \left\{\sum_{m=1}^{\infty} \frac{\lambda^{m}}{m} \sum_{x \in \mathrm{fix}\left(R_{m}\right)} \frac{1}{R_{m}^{\prime}(x)}\right\} .
\end{aligned}
$$

The first factor in (3.3) is the Fredholm determinant of the operator

$$
\left(L_{1} g\right)(x)=\sum_{R y=x} \frac{g(y)}{R^{\prime}(y)}
$$

the second one is the Ruelle $\zeta$-function (Ruelle [17]). In the case when $R$ is a polynomial, the operator $L_{1}$ is a Volterra operator.

We observe that

$$
\left(L_{1}^{*} f\right)(z)=f(R(z))
$$

\section{Calculation of $D(\lambda)$ with the Help of Perturbation Theory}

4.1. In order to prevent long calculations, we assume that the function $R$ obey the following conditions:

(a) $\forall c \in \operatorname{crit}(R), R^{\prime \prime}(c) \neq 0$;

(b) $\forall c, c^{\prime} \in \operatorname{crit}(R), \forall n \in \mathbb{N}, R_{n}(c) \neq c^{\prime}$.

Remark. For polynomials with real Julia sets the above conditions are satisfied automatically. Indeed, let $R$ be such a polynomial. If $x \in J$, then all roots of the equation $R(y)=x$ are real numbers. Hence $R(\bar{z})=\overline{R(z)}$, for all $z \in \mathbb{C}$. If $u(z)$ is the Green function of the domain $G=\mathbb{C} \backslash J$ with the pole at infinity, then an open set $\{u(z)<a\}, a>0$, is symmetric with respect to the real axis $\mathbb{R}$ and all its components contain points of $J$. It follows from this $\operatorname{crit}(R) \subset \mathbb{R}$. Suppose that $R^{\prime \prime}(c)=0$, for some $c \in \operatorname{crit}(R)$. Then the set $\{u(z)<u(c)\}$ consists of more than two components. One of them does not intersect $\mathbb{R}$. So there are points of $J$ outside of $\mathbb{R}$. This contradiction proves (a). In its turn, (a) implies (b), if we apply (a) to the iterations.

4.2. Let us introduce a space $A^{*}(\Omega, R)$ of functions: $f \in A^{*}(\Omega, R)$ iff $f$ is defined and holomorphic function in a domain $\Omega_{f}$, which contains $\overline{\mathbb{C}} \backslash \Omega$ minus all preimages of the set crit $(R)$ under the iterations $R_{n}, n=0,1,2, \ldots$, and $f(\infty)=0$. We regard that 
$A^{*}(\Omega) \subset A^{*}(\Omega, R)$. Define the operator $L^{*}$ in the space $A^{*}(\Omega, R)$ by the formula (2.6) (we preserve the symbol $L^{*}$ for this operator). $L^{*} f$ is a Cauchy-type integral, hence $L^{*}: A^{*}(\Omega, R) \rightarrow A^{*}(\Omega)$. Then the operator $L^{*}$ considered in the spaces $A^{*}(\Omega, R)$ and $A^{*}(\Omega)$ has the same eigenvalues with the same multiplicities. Define now an operator $K$ in the space $A^{*}(\Omega, R)$ :

$$
(K f)(z)=\frac{f(R(z))}{R^{\prime}(z)}, \quad f \in A^{*}(\Omega, R)
$$

Because of (2.7), we shall consider the operator $L^{*}$ as a finite-dimensional perturbation of the operator $K$, which, in its turn, by (3.4), is a slight variant of the operator $L_{1}^{*}$.

First of all, we study the spectrum of the operator $K$. We restrict our attention to case (2.1): $\sigma \neq \infty$ [in case (2.2) of a superattracting point similar considerations prove that the operator $K$ is a Volterra operator].

Let $\Omega^{*}$ be a small enough neighbourhood of infinity, invariant under $R$. We consider the operator $K$ in the space $A_{0}\left(\Omega^{*}\right)$. It is easy to see that the spectrum of $K$ does not change this replacement.

Use the change of variables (2.3). If a function $h(u)$ is analytic in a neighbourhood of infinity and $h(\infty)=0$, then $f(z)=h(\varphi(z)) \in A_{0}\left(\Omega^{*}\right)$, and

$$
(K h)(u)=\frac{h(\sigma u)}{R^{\prime}(z)} .
$$

Let us introduce the function $z=\psi(u)$, inverse to $\varphi(z)$, then $R(z)=\psi(\sigma \varphi(z))$, hence

$$
R^{\prime}(z)=\sigma \psi^{\prime}(\sigma u) \varphi^{\prime}(z)=\frac{\sigma \psi^{\prime}(\sigma u)}{\psi^{\prime}(u)} .
$$

If we substitute (4.3) in (4.2), then we obtain

$$
K h(u)=\frac{1}{\sigma} \frac{h(\sigma u)}{\psi^{\prime}(\sigma u)} \psi^{\prime}(u)
$$

The functions $\left\{1 / u^{n}\right\}_{n=0}^{\infty}$ are eigenfunctions of the operator $h(u) \mapsto \frac{h(\sigma u)}{\sigma}$, therefore the functions $\left\{\psi^{\prime}(u) / u^{n}\right\}_{n=1}^{\infty}$ form eigenfunctions of the considered operator $K$ :

$$
K\left[\frac{\psi^{\prime}(u)}{u^{n}}\right]=\frac{1}{\sigma^{n+1}} \frac{\psi^{\prime}(u)}{u^{n}}, \quad u=\varphi(z) .
$$

Since the latter set of eigenfunctions is complete in the space $A_{0}\left(\Omega^{*}\right)$, then the spectrum of the operator $K$ is simple and consists of the points $\left\{1 / \sigma^{n+1}\right\}_{n=1}^{\infty}$.

This fact follows also from the examination of Neumann series. Indeed, we have, for $f \in A^{*}(\Omega, R), z \in \Omega_{f}$ and sufficiently large $N$ :

$$
\begin{aligned}
\sum_{n=0}^{\infty}\left(\lambda^{n} K^{n}\right) f(z) & =\sum_{n=0}^{\infty} \frac{\lambda^{n} f\left(R_{n}(z)\right)}{R_{n}^{\prime}(z)} \\
& =\sum_{n=0}^{N-1} \frac{\lambda^{n} f\left(R_{n}(z)\right)}{R_{n}^{\prime}(z)}+\frac{\lambda^{N}}{R_{N}^{\prime}(z)} \psi(u) \sum_{n=0}^{\infty} \frac{\lambda^{n}}{\sigma^{n}}\left(\frac{f \circ \psi}{\psi^{\prime}}\right)\left(\sigma^{n} u\right) \\
& =\sum_{n=0}^{N-1} \frac{\lambda^{n} f\left(R_{n}(z)\right)}{R_{n}^{\prime}(z)}+\frac{\lambda^{N} \psi(u)}{R_{N}^{\prime}(z)} \sum_{l=1}^{\infty} \frac{c_{l}}{1-\frac{\lambda}{\sigma^{l+1}}} \frac{1}{u^{l}},
\end{aligned}
$$


where $u=R_{N}(z)$, and numbers $c_{l}, l=1, \ldots$, are defined by the expansion $\frac{f \circ \psi}{\psi^{\prime}}(u)$ $=\sum_{l=1}^{\infty} \frac{c_{l}}{u^{l}}$ at infinity. Thus, the points $\left\{1 / \sigma^{l+1}\right\}_{l=1}^{\infty}$ are the poles of the resolvent $(I-\lambda K)^{-1}$ and form the spectrum of the operator $K$. In particular,

$$
\operatorname{det}(I-\lambda K)=\prod_{n=1}^{\infty}\left(1-\frac{\lambda}{\sigma^{n+1}}\right) .
$$

Let us now continue (2.7) using conditions (a) and (b):

$$
\left(L^{*} f\right)(z)=\frac{f(R(z))}{R^{\prime}(z)}-\sum_{c \in \mathrm{crit}(R)} \frac{f(R(c))}{R^{\prime \prime}(c)} \frac{1}{z-c} .
$$

In other words,

$$
L^{*}=K-F G,
$$

where $G$ and $F$ are the operators from $A^{*}(\Omega, R)$ to $\mathbb{C}^{l}$ and from $\mathbb{C}^{l}$ to $A^{*}(\Omega, R)$ respectively, $l=$ card $\operatorname{crit}(R)$ :

$$
\begin{gathered}
G f=\left\{\frac{f(R(c))}{R^{\prime \prime}(c)}\right\}_{c \in \operatorname{crit}(R)}, \quad f \in A^{*}(\Omega, R), \\
(F \alpha)(z)=\sum_{c \in \operatorname{crit}(R)} \frac{\alpha_{c}}{z-c}, \quad \alpha \in \mathbb{C}^{l} .
\end{gathered}
$$

By (4.9), we have

$$
D(\lambda)=\operatorname{det}\left(I-\lambda L^{*}\right)=\operatorname{det}(I-\lambda K) \operatorname{det} M(\lambda),
$$

where

$$
M(\lambda)=1+\lambda G(I-\lambda K)^{-1} F
$$

is an operator taking $\mathbb{C}^{l}$ into $\mathbb{C}^{l}$.

Really,

$$
\begin{aligned}
\operatorname{det}\left(I-\lambda L^{*}\right) & =\operatorname{det}(I-\lambda K+\lambda F G)=\operatorname{det}(I-\lambda K) \operatorname{det}\left(I+\lambda(I-\lambda K)^{-1} F G\right) \\
& =\operatorname{det}(I-\lambda K) \operatorname{det}\left(1+\lambda G(I-\lambda K)^{-1} F\right)
\end{aligned}
$$

(the latter equality follows from the definition of the determinant).

Now we use (4.1), (4.10), (4.11), and (4.13) and get

$$
\begin{aligned}
M(\lambda) & =1+\lambda G\left(\sum_{n=0}^{\infty} \lambda^{n} K^{n}\right) F=1+\lambda G\left(\sum_{n=0}^{\infty} \frac{\lambda^{n}}{R_{n}^{\prime}(z)\left(R_{n}(z)-c_{j}\right)}\right)_{j=1}^{l} \\
& =1+\left\|\sum_{n=0}^{\infty} \frac{\lambda^{n+1}}{R^{\prime \prime}\left(c_{i}\right) R_{n}^{\prime}\left(R\left(c_{i}\right)\right)\left[R_{n+1}\left(c_{i}\right)-c_{j}\right]}\right\|_{i, j=1}^{l} \\
& =1+\left\|\sum_{n=1}^{\infty} \frac{\lambda^{n}}{R_{n}^{\prime \prime}\left(c_{i}\right)\left[R_{n}\left(c_{i}\right)-c_{j}\right]}\right\|_{i, j=1}^{l}
\end{aligned}
$$

(symbol $\|\cdot\|_{i, j=1}^{l}$ denotes a square matrix $l \times l$ ). 
Finally, using (4.14), (4.7), and (4.12), we obtain the desired equality

$$
D(\lambda)=\prod_{n=1}^{\infty}\left(1-\frac{\lambda}{\sigma^{n+1}}\right) \operatorname{det}\left[1+\left\|\sum_{n=1}^{\infty} \frac{\lambda^{n}}{R_{n}^{\prime \prime}\left(c_{i}\right)\left[R_{n}\left(c_{i}\right)-c_{j}\right]}\right\|_{i, j=1}^{l}\right]
$$

or, equivalently, $\zeta(\lambda)\left(1-\frac{\lambda}{\sigma}\right)=\operatorname{det}\left[1+\left\|\sum_{n=1}^{\infty} \frac{\lambda^{n}}{R_{n}^{\prime \prime}\left(c_{i}\right)\left[R_{n}\left(c_{i}\right)-c_{j}\right]}\right\|_{i, j=1}^{l}\right]$.

\section{Calculation of the Resolvent Function $E(x, z ; \lambda)$}

Recall, that

$$
E(x, z ; \lambda)=(I-\lambda L)^{-1} \frac{1}{z-x}=\left(I-\lambda L^{*}\right)^{-1} \frac{1}{z-x}
$$

(where the operator $L$ acts on the variable $x \in \Omega$, and the operator $L^{*}$ acts on the variable $\left.z \in \Omega^{*}\right)$.

By (4.9) we have

$$
\begin{aligned}
& \left(I-\lambda L^{*}\right)^{-1}=(I-\lambda K+\lambda F G)^{-1} \\
& \quad=(I-\lambda K)^{-1}-\lambda(I-\lambda K)^{-1} F M^{-1}(\lambda) G(I-\lambda K)^{-1}
\end{aligned}
$$

(the last equality is checked directly); in (5.2), as above, we set

$$
M(\lambda)=1+\lambda G(I-\lambda K)^{-1} F: \mathbb{C}^{l} \rightarrow \mathbb{C}^{l} .
$$

Let

$$
H(x, z ; \lambda)=(I-\lambda K)^{-1} \frac{1}{z-x}=\sum_{n=0}^{\infty}\left(\lambda^{n} K^{n}\right) \frac{1}{z-x}=\sum_{n=0}^{\infty} \lambda^{n} \frac{1}{R_{n}^{\prime}(z)\left[R_{n}(z)-x\right]} .
$$

From Eqs. (5.1)-(5.3) we obtain the required formula

$$
\begin{aligned}
E(x, z ; \lambda) & =H(x, z ; \lambda)-\lambda\left(\frac{H\left(c_{1}, z ; \lambda\right)}{R^{\prime \prime}\left(c_{1}\right)}, \ldots, \frac{H\left(c_{l}, z ; \lambda\right)}{R^{\prime \prime}\left(c_{l}\right)}\right) \\
& \times M^{-1}(\lambda)\left[\begin{array}{c}
H\left(x, R\left(c_{1}\right) ; \lambda\right) \\
\vdots \\
H\left(x, R\left(c_{l}\right) ; \lambda\right)
\end{array}\right] .
\end{aligned}
$$

It should be noted by $(4.6)$ the function $H(\cdot, \cdot ; \lambda)$ is a meromorphic function in $\mathbb{C}$ with poles in the points $\left\{\sigma^{n+1}\right\}_{n=1}^{\infty}$ (cf. Fatou [9]), and that

$$
M(\lambda)=1+\lambda\left\|\frac{H\left(c_{i}, R\left(c_{j}\right) ; \lambda\right)}{R^{\prime \prime}\left(c_{i}\right)}\right\|_{i, j=1}^{l} .
$$

The eigenfunctions of the operators $L$ and $L^{*}$ can be explicitly expressed in terms of the function $H$.

\section{Example 1: $R(z)=z^{2}-p, p>2$}

In this case the obtained formulae (4.15) and (5.4) are simplified as the unique critical point of the polynomial $R$ is the point $z=0$, and $R_{n}^{\prime}(z)=2^{n} R_{n-1}(z) \ldots R(z) z$. 
Therefore

$$
\begin{gathered}
D(\lambda)=1+\sum_{n=1}^{\infty} \frac{(\lambda / 2)^{n}}{R(0) R_{2}(0) \ldots R_{n}(0)}, \\
H(x, z ; \lambda)=\sum_{n=0}^{\infty} \frac{(\lambda / 2)^{n}}{z R(z) \ldots R_{n-1}(z)\left[R_{n}(z)-x\right]}, \\
E(x, z ; \lambda)=H(x, z ; \lambda)-\frac{\lambda}{2} \frac{H(0, z ; \lambda) H(x, R(0) ; \lambda)}{D(\lambda)} .
\end{gathered}
$$

\section{Example 1: Continuation. Calculation of the Taylor Expansion} of the Function $1 / D(\lambda)$

Using the Neumann series, we obtain another expression for the function $E$. We have:

$$
E(x, z ; \lambda)=(I-\lambda L)^{-1} \frac{1}{z-x}=\sum_{n=0}^{\infty} \lambda^{n} L^{n} \frac{1}{z-x} .
$$

Let us investigate the function $L^{n} \frac{1}{z-x}$. For this purpose we need some information about orthogonal polynomials (Akhiezer [1]) and, in particular, about orthogonal polynomials with respect to the balanced measure $\mu$ of the polynomial $R(z)$ (the measure $\mu$ was discovered by Brolin [8]. Orthogonal polynomials with respect to $\mu$ were investigated by Pitcher and Kinney [16], Bellissard, Bessis, Moussa [3], Barnsley, Geronimo, Harrington [2], Bessis and Moussa [5]; see also Bessis, Mehta, and Moussa [4] and Sodin, Yuditski [19]).

Let $S$ be a polynomial of a degree $m$. Hereafter the polynomial $S$ is an iteration of the quadratic polynomial $x^{2}-p$, more generally, the arbitrary monic centered polynomial

$$
S(x)=x^{m}+a_{m-2} x^{m-2}+\ldots+a_{1} x+a_{0} .
$$

Then

$$
L_{S} \frac{1}{z-x} \equiv \sum_{S y=x} \frac{1}{\left[S^{\prime}(y)\right]^{2}} \frac{1}{z-y}=\frac{Q_{m-1}(z, x)}{S(z)-x},
$$

where $Q_{m-1}(z, x)$ is a polynomial on variable $z$ of degree $m-1$. The values of this polynomial in the points $y \in S_{-1}(x)$ are equal to $\frac{1}{S^{\prime}(y)}$. This implies that the polynomial $Q_{m-1}(z, x)$ is an orthogonal one to the powers $z^{k}, 0 \leqq k \leqq m-2$, with respect to the probability measure $\lambda_{x}$ uniformly distributed at the points of the set $S_{-1}(x)$. Indeed,

$$
\int z^{k} Q_{m-1}(z, x) d \lambda_{x}(z)=\frac{1}{m} \sum_{S y=x} y^{k} Q_{m-1}(y, x)=\frac{1}{m} \sum_{S y=x} \frac{y^{k}}{S^{\prime}(y)}=0
$$

for $0 \leqq k \leqq m-2$, since the last sum is equal to the sum of finite residues of the rational function $\frac{y^{k}}{S(y)}$. 
Let $P_{k}, 0 \leqq k \leqq m-1, \operatorname{deg} P_{k}=k$, be orthonormal polynomials with respect to the measure $\lambda_{x}$. Then $Q_{m-1}=\beta P_{m-1}$, where $\beta$ is a constant, which will be calculated later on.

The polynomials $P_{k}$ satisfy a three-term recursion relation as follows:

$$
b_{k+1} P_{k+1}(z)=\left(z-a_{k}\right) P_{k}(z)-b_{k} P_{k-1}(z), \quad k \leqq m-2,
$$

$a_{k}=a_{k}(x), b_{k}=b_{k}(x)$.

We join the polynomial $P_{m}(z)=S(z)-x$ to the system $\left\{P_{k}\right\}, 0 \leqq k \leqq m-1$. Then (7.3) holds for $k=m-1$, moreover

$$
b_{m}=\left(b_{1} \ldots b_{m-1}\right)^{-1} \text {. }
$$

The corresponding polynomial of the second kind is equal to

$$
\int \frac{P_{m}(z)-P_{m}(u)}{z-u} d \lambda_{x}(u)=\frac{1}{m} \sum_{S(y)=x} \frac{S(z)-x}{z-y}=\frac{1}{m} S^{\prime}(z) .
$$

Therefore (see, for example, Akhiezer [1, Chap. 1])

$$
\frac{S^{\prime}(z)}{m(S(z)-x)}=\frac{1}{z-a_{1}-\frac{b_{1}^{2}}{z-a_{2}-\frac{b_{2}^{2}}{\vdots}}} .
$$

Besides, it follows readily from (7.3) that

$$
\frac{P_{m-1}(z)}{b_{m}(S(z)-x)}=\frac{1}{z-a_{m-1}-\frac{b_{m-1}^{2}}{z-a_{m-2}-\frac{b_{m-2}^{2}}{\vdots}}} .
$$

Now we shall calculate the constant $\beta$. The leading coefficient of the polynomial $Q_{m-1}(z, x)$ is equal to

$$
\lim _{z \rightarrow \infty} z \frac{Q_{m-1}(z, x)}{S(z)-x}=\left(L_{S} 1\right)(x)=\sum_{S(y)=x} \frac{1}{\left[S^{\prime}(y)\right]^{2}}=m \int Q_{m-1}^{2}(z, x) d \lambda_{x}(z)=m \beta^{2} .
$$

On the other hand, it is equal to the leading coefficient of the polynomial $P_{m-1}$ multiplied by $\beta$, that is [by (7.3)] it is equal to

$$
\frac{\beta}{b_{1} \ldots b_{m-1}} \text {. }
$$

Thus,

$$
m \beta^{2}=\frac{\beta}{b_{1} \ldots b_{m-1}},
$$


or, using (7.4), we obtain

$$
\beta=\frac{1}{m b_{1} \ldots b_{m-1}}=\frac{b_{m}}{m} .
$$

Hence Eq. (7.6) we can rewrite in the following form:

$$
\frac{Q_{m-1}(z, x)}{S(z)-z}=\frac{b_{m}^{2}}{m} \frac{1}{z-a_{m-1}-\frac{b_{m-1}^{2}}{z-a_{m-2}-\frac{b_{m-2}^{2}}{\vdots}}} .
$$

Let now $\mu$ be the balanced measure of the polynomial $R, S=R_{n}$ and $x=0$. The polynomial $R_{n}$ is orthogonal to the powers $z^{k}, 0 \leqq k \leqq 2^{n}-1$, with respect to the measure $\mu$, hence as it follows from (7.5) the numbers $b_{k}^{2}=b_{k}^{2}(0)$ is the sequence of coefficients in the continued fraction expansion of the Stieltjes transformation $\int \frac{d \mu(\tau)}{z-\tau}$, and $a_{k}=a_{k}(0)=0$.

We denote by $\omega_{n}$ the rational function

$$
\omega_{n}(\mathrm{z})=\frac{\mathrm{P}_{2^{n}-1}(z)}{b_{2^{n}} P_{2^{n}}(z)}=\frac{\sqrt{p}}{b_{2^{n}}} \frac{P_{2^{n}-1}(z)}{R_{n}(z)}
$$

where $\left(P_{k}\right)_{k=0}^{\infty}$ is the system of orthonormal polynomials with respect to the measure $\mu$.

Then using Eqs. (6.3), (7.1), (7.2), (7.8) (with $x=0, m=2^{n}, S=R_{m}$ ) and, at last, (7.9), we obtain the required formula

$$
E(0, z ; \lambda)=\frac{H(0, z ; \lambda)}{D(\lambda)}=\sum_{n=0}^{\infty}\left(\frac{\lambda}{2}\right)^{n} b_{2^{n}}^{2} \omega_{n}(z)
$$

Calculating the residues at the point $z=\infty$ of each part of (7.10), we obtain finally

$$
\frac{1}{D(\lambda)}=\sum_{n=0}^{\infty} b_{2^{n}}^{2}\left(\frac{\lambda}{2}\right)^{n}
$$

Remark. Similar formulae can be written for every monic centered polynomial, which satisfies the conditions (a)-(b) (see Sect. 4.1).

Comparing (6.1), (7.11), and (3.3) we get the interesting identities

$$
1+\sum_{n=1}^{\infty} \frac{\lambda^{n}}{R(0) \ldots R_{n}(0)}=\frac{1}{\sum_{n=0}^{\infty} b_{2^{n}}^{2} \lambda^{n}}=\exp \left\{\sum_{m=1}^{\infty} \frac{\lambda^{m}}{\mathrm{~m}} \sum_{x \in \mathrm{fix}\left(R_{m}\right)} \frac{1}{x R(x) \ldots R_{m-1}(x)}\right\}
$$


8. Example 2: $R(z)=\sigma z-\frac{1}{z}, 1<\sigma<\infty$

The upper and lower halfplanes as well as the real axis are invariant under the map $R$. Hence $J \subset \mathbb{R}$ and Cantorian (since $R$ is expanding, if $\sigma>1$ ). The function $R$ has two symmetric critical points $c_{1}=c=\frac{i}{\sqrt{\sigma}}, c_{2}=-c$. Besides, for all $n \in \mathbb{N}$ the functions $R_{n}$ and $R_{n}^{\prime \prime}$ are odd functions.

We use (4.14) and obtain

$$
\begin{aligned}
\operatorname{det} M(\lambda) & =\left|\begin{array}{cc}
1+\sum_{n=1}^{\infty} \frac{\lambda^{n}}{R_{n}^{\prime \prime}(c)\left[R_{n}(c)-c\right]}, & \sum_{n=1}^{\infty} \frac{\lambda^{n}}{R_{n}^{\prime \prime}(c)\left[R_{n}(c)+c\right]} \\
\sum_{n=1}^{\infty} \frac{\lambda^{n}}{R_{n}^{\prime \prime}(c)\left[R_{n}(c)+c\right]}, & 1+\sum_{n=1}^{\infty} \frac{\lambda^{n}}{R_{n}^{\prime \prime}(c)\left[R_{n}(c)-c\right]}
\end{array}\right| \\
& =\left(1+2 c \sum_{n=1}^{\infty} \frac{\lambda^{n}}{R_{n}^{\prime \prime}(c)\left[R_{n}^{2}(c)-c^{2}\right]}\right)\left(1+2 \sum_{n=1}^{\infty} \frac{\lambda^{n} R_{n}(c)}{R_{n}^{\prime \prime}(c)\left[R_{n}^{2}(c)-c^{2}\right]}\right) .
\end{aligned}
$$

Since $R$ is expanding, the function $\operatorname{det} M(\lambda)$ has a root $\lambda_{1}$ with least modulus, and $\lambda_{1}>0$, and for any point $x \in J \sum_{R_{n}(y)=x} \frac{1}{\left|R_{n}^{\prime}(y)\right|^{2}} \asymp \frac{c}{\lambda_{1}^{n}}, c=c(x)>0$.

Let us find bounds for $\lambda_{1}$. If $a_{\sigma}=\frac{1}{\sqrt{\sigma-1}}$ is the positive repulsive fixed point of the function $R$, then $J \subset\left[-a_{\sigma}, a_{\sigma}\right]$, and $\left|R^{\prime}\right|_{J} \geqq R^{\prime}\left(a_{\sigma}\right)=2 \sigma-1$, hence $\left|R_{n}^{\prime}\right|_{J} \geqq(2 \sigma-1)^{n}$, and

$$
\sum_{R_{n}(y)=x} \frac{1}{\left|R_{n}^{\prime}(y)\right|^{2}} \leqq \frac{2^{n}}{(2 \sigma-1)^{2 n}} .
$$

This inequality implies $\lambda_{1} \geqq \frac{(2 \sigma-1)^{2}}{2}$.

On the other hand, the value $\log \frac{1}{\lambda_{1}}$ is equal to the pressure of the function $-2 \log \left|R^{\prime}\right|$ (Bowen[7, Chap. 1]). Let us consider the Dirac measure $\varepsilon$ concentrated at the fixed point $a_{\sigma}$, and use the variational principle (Bowen [7, Chap. 1]):

$$
\log \frac{1}{\lambda_{1}}>\int\left(-2 \log \left|R^{\prime}\right|\right) d \varepsilon=-2 \log (2 \sigma-1)
$$

that is $\lambda_{1}<(2 \sigma-1)^{2}$.

Thus, we have proved that $\frac{(2 \sigma-1)^{2}}{2} \leqq \lambda_{1}<(2 \sigma-1)^{2}$.

In particular, for $\sigma>\frac{2+\sqrt{2}}{2}$ the least root $\lambda_{1}$ of the function $\operatorname{det} M(\lambda)$ lies outside of the circle of convergence $\left\{\lambda:|\lambda|<\sigma^{2}\right\}$ of the Taylor expansion of this function. 


\section{Conclusion}

Our method works, when $R$ is an expanding rational function and a weight $\phi$ in the Ruelle operator is a rational function with the poles outside of $J$ (the Julia set $J$ is not necessarily a subset of the real axis). Then one can write down an explicit expression for the Fredholm determinant of the operator

$$
(L g)(x)=\sum_{R(y)=x} g(y) \phi(y),
$$

acting in a space of functions $g$ analytic in a neighbourhood of $J$. For example, let $R$ be a finite Blaschke product and $J$ be the unit circle $S_{1}=\{|z|=1\}$. Consider $\phi(z)$ $=\left|R^{\prime}(x)\right|^{-2}$, for $z \in S_{1}$. This function extends to a rational function according to the formula $\phi(z)=\left(R(z) / z R^{\prime}(z)\right)^{2}$.

The approach suggested at the present paper for the calculation of the Fredholm determinant is applied also to the essentially more general situations, namely, when the weight $\phi$ is a holomorphic function in some neighbourhood of bounded Julia set of an expanding rational function. In particular, the operators

$$
\left(L_{s} g\right)(x)=\sum_{R(y)=x} \frac{g(y)}{\left|R^{\prime}(y)\right|^{s}}
$$

$\left(R(z)=z^{2}-p, p>2, s \in \mathbb{R}\right)$ are related to this case. The authors will return to this question in their coming paper.

Acknowledgements. We thank A. Eremenko, A. Grishin, M. Lyubich, and L. Pastur for valuable discussions.

This paper was completed while the first named author was a guest of the Sonderforschungsbereich 170. The first named author would like to thank Professor M. Denker and his colleagues for their generous hospitality.

\section{References}

1. Akhiezer, N.I.: Classical problem of moments. Moscow: GIFML 1961

2. Barnsley, M., Geronimo, J., Harrington, A.: Orthogonal polynomials associated with invariant measures on Julia sets. Bull. Am. Math. Soc. 7, 381-384 (1982)

3. Bellissard, J., Bessis, D., Moussa, P.: Chaotic states for almost periodic Schrödinger operators. Phys. Rev. Lett. 49, 701-704 (1982)

4. Bessis, D., Mehta, M.L., Moussa, P.: Orthogonal polynomials on a family of Cantor sets and the problem of iterations of quadratic mappings. Lett. Math. Phys. 6, 123-140 (1982)

5. Bessis, D., Moussa, P.: Orthogonality properties of iterated polynomial mappings. Commun. Math. Phys. 88, 503-529 (1983)

6. Blanchard, P.: Complex analytic dynamics on the Riemann sphere. Bull. Am. Math. Soc. 11, 85-141 (1984)

7. Bowen, R.: Equilibrium states and the ergodic theory of Anosov diffeomorphisms. Berlin, Heidelberg, New York: Springer 1975

8. Brolin, H.: Invariant sets under iteration of rational functions. Arkiv Matematik 6, 103-144 (1965)

9. Fatou, P.: Ann. Scient. Ecole Norm. Sup. (3) 27, 43-53 (1910)

10. Golysin, G.M.: Geometric function theory in complex variable. Moscow 1966

11. Hirsch, M., Pugh, C.: Stable manifolds and hyperbolic sets. Global Analysis, Proc. Symp. in Pure Math. 14, Providence, R.I.: Am. Math. Soc. 1970, pp. 133-163

12. Kadanoff, L., Tang, C.: Escape from strange repellers. Proc. Nat. Acad. Sci. USA 81, 1276-1279 (1984) 
13. Lyubich, M.: Dynamics of rational transformations: topological picture. Russ. Math. Surv. 41, 43-117 (1986)

14. Levin, G.M.: Polynomial Julia sets and Padé approximations. Unpublished manuscript (1988)

15. Milnor, J.: Dynamics in one complex variable: introductory lectures. Preprint, Stony Brook $1990 / 5$

16. Pitcher, T.S., Kinney, J.R.: Some connections between ergodic theory and the iteration of polynomials. Arkiv Matematik 8, 25-32 (1969)

17. Ruelle, D.: Zeta functions for expanding maps and Anosov flows. Invent. Math. 34, 231-242 (1976)

18. Ruelle, D.: The thermodynamics formalism for expanding maps. Commun. Math. Phys. 125, 239-262 (1989)

19. Sodin, M., Yuditski, P.: The limit-periodic finite-difference operator on $l^{2}(\mathbb{Z})$ associated with iterations of quadratic polynomials. J. Stat. Phys. 60, 863-873 (1990)

20. Sullivan, D.: Quasiconformal homeomorphisms and dynamics. I. Solution of the Fatou-Julia problem on wandering domains. Ann. Math. 122, 401-418 (1985)

21. Widom, N., Bensimon, D., Kadanoff, L., Shenker, S.: Strange objects on the complex plane. J. Stat. Phys. 32, 443-454 (1983)

Communicated by J.-P. Eckmann 